\title{
In Vitro Selection of a New Lanthanide-Dependent DNAzyme for Ratiometric Sensing Lanthanides
}

\author{
Po-Jung Jimmy Huang, Mahsa Vazin and Juewen Liu* \\ Department of Chemistry, Waterloo Institute for Nanotechnology \\ University of Waterloo \\ 200 University Ave West, Waterloo, Ontario, Canada, N2L 3G1 \\ Fax: (+1) 519-746-0435 \\ E-mail:liujw@uwaterloo.ca
}

This document is the Accepted Manuscript version of a Published Work that appeared in final form in Analytical Chemistry, copyright (C) American Chemical Society after peer review and technical editing by publisher. To access the final edited and published work see Huang, P.-J. J., Vazin, M., \& Liu, J. (2014). In Vitro Selection of a New LanthanideDependent DNAzyme for Ratiometric Sensing Lanthanides. Analytical Chemistry, 86(19), 9993-9999. https://doi.org/10.1021/ac5029962 
Abstract. Developing biosensors for lanthanides is an important but challenging analytical task. To address this problem, in vitro selection of RNA-cleaving DNAzymes was carried out using a library containing a region of 35 random nucleotides in the presence of $\mathrm{Lu}^{3+}$, since $\mathrm{Lu}^{3+}$ was reported to be the most efficient lanthanide for RNA cleavage. The resulting DNA sequences can be aligned to a single family with two conserved stretches of nucleotides. One of the representative DNAzymes (named Lu12) was further studied. Lu12 is more active with smaller lanthanides and has the lowest activity in the presence of the largest lanthanide $\left(\mathrm{Lu}^{3+}\right)$. Its cleavage rate is $0.12 \mathrm{~min}^{-1}$ in the presence of $10 \mu \mathrm{M} \mathrm{Nd} \mathrm{d}^{3+}$ at $\mathrm{pH}$ 6.0. This is a new DNAzyme and a catalytic beacon sensor is designed by attaching a fluorophore/quencher pair, detecting $\mathrm{Nd}^{3+}$ down to $0.4 \mathrm{nM}$ (72 parts-per-trillion). This DNAzyme is highly selective for lanthanides as well, showing cleavage only with two non-lanthanide ions: $\mathrm{Y}^{3+}$ and $\mathrm{Pb}^{2+}$. We previously reported a DNAzyme named Ce13d, which has similar responses to all the trivalent lanthanides. Combining these two allows for a ratiometric assay that identifies a few large lanthanides. 


\section{Introduction}

The lanthanides are the 15 elements in the first row of the $f$-block in the periodic table. Due to their unique electronic, optical, magnetic and catalytic properties, lanthanides are extremely important for modern technological applications and are called industrial vitamins. ${ }^{1,2}$ The consumption of lanthanides currently reaches $\sim 100,000$ metric tons per year, ${ }^{3}$ calling for analytical tools for monitoring pollution, recycling, and finding new mineral sources. Currently, lanthanide analysis mainly relies on instrumentation methods such as inductively coupled plasma mass spectrometry (ICP-MS), ${ }^{4}$ which is costly, cumbersome for sample preparation, and available only in large centralized labs. In this regard, sensors can provide complementary analytical information for on-site and real-time detection.

While a few optical and electrochemical sensors for lanthanides were reported based on small molecule chelators, they suffer from poor selectivity and low sensitivity. ${ }^{5-7}$ There are two levels of selectivity requirement. First, a sensor needs to tell whether an analyte is a lanthanide or not. Second, it is even more challenging to tell its identity from a group of 15 . Chemical probing of lanthanides has been a long-standing challenge. This is attributed to that lanthanides only differ by the number of inner $4 f$ electrons, which do not readily participate in chemical bonding and are shielded by the filled $4 d, 5 s$ and $5 p$ orbitals. In addition, all the trivalent lanthanides have the same charge and similar sizes, displaying extremely similar chemical properties. With 15 of them in the group, limited success was achieved using rational ligand design.

We reason that DNA might be a useful platform for sensing lanthanides. DNA contains phosphate groups that bind lanthanides with high affinity. At the same time, the bases provide nitrogen ligands, which might be useful for discrimination within the group. ${ }^{8}$ Lanthanides form complexes with nucleotides, especially with adenosine or guanosine phosphates. ${ }^{9,} 10 \mathrm{GT}$ rich DNA is effective in 
sensitized $\mathrm{Tb}^{3+}$ luminescence. ${ }^{11}{ }^{12}$ Lanthanides have also been used as chemical and optical probes for DNA, ${ }^{13,14}$ and can cleave RNA. ${ }^{15}$ It is however still difficult to rationally design DNA sequences that can selectively bind lanthanides; many other metals also have high affinity for DNA.

DNAzymes are DNA-based catalysts, where metal ion cofactors are required for activity. ${ }^{16-21}$ In the past 15 years, RNA-cleaving DNAzymes have emerged to be a unique platform for metal detection. ${ }^{22-28}$ Using in vitro selection, DNAzymes specific for $\mathrm{Pb}^{2+},{ }^{29} \mathrm{Zn}^{2+},{ }^{30} \mathrm{Cu}^{2+},{ }^{31} \mathrm{UO}_{2}{ }^{2+},{ }^{32}$ and $\mathrm{Hg}^{2+}$ were isolated. ${ }^{33} \mathrm{~A}$ few DNAzymes involving lanthanides were also reported. For example, DNAzymes for DNA cleavage were selected in the presence of $\mathrm{Ce}^{3+}, \mathrm{Eu}^{3+}$ or $\mathrm{Yb}^{3+}$ together with $\mathrm{Zn}^{2+} .{ }^{34} \mathrm{~A} \mathrm{Mg}^{2+}-$ dependent DNA ligase and a small $\mathrm{Pb}^{2+}$-dependent ribozyme are accelerated by lanthanides. ${ }^{35,36}$ On the other hand, $\mathrm{Tb}^{3+}$ inhibits the 8-17 DNAzyme, ${ }^{13}$ and the hammerhead ribozyme. ${ }^{37}$ The first DNAzyme, GR5, was also found to be active with lanthanides alone, although the rate was quite slow (below 0.02 $\left.\min ^{-1}\right) \cdot{ }^{38}$

We recently selected a DNAzyme (named Ce13d) using $\mathrm{Ce}^{4+}$ as the intended target. ${ }^{39}$ Interestingly, this enzyme is similarly active with all the trivalent lanthanides but has almost no activity with $\mathrm{Ce}^{4+}$. This is attributed to that $\mathrm{Ce}^{4+}$ is a poor metal cofactor for cleaving RNA. ${ }^{40}$ Since $\mathrm{Ce}^{4+}$ is a strong oxidant, we suspect that a small fraction ( $1 \%$ by our estimation) of it was converted to $\mathrm{Ce}^{3+}$, which became the active metal in our previous selection.

Ce13d provides a starting point for solving the lanthanide detection problem since it has excellent selectivity for lanthanides as a group. However, it has little discrimination within the group. Herein, we aim to test whether we can obtain new DNAzymes that better discriminate each lanthanide. If so, by combining these two DNAzymes, a ratiometric sensor may be developed, providing information about individual ions within this series. 


\section{Materials and Methods}

Chemicals. The DNA library for in vitro selection, related primers and fluorophore/quencher modified DNAs were purchased from Integrated DNA Technologies (IDT, Coralville, IA). The sequences of DNA used in this selection are listed in Table S1. The trans-cleaving enzyme strands and their mutants were purchased from Eurofins (Huntsville, AL). The lanthanides and other metals were purchased from Sigma-Aldrich. Tris(hydroxymethyl)aminomethane (Tris), 2-(N-morpholino)ethanesulfonic acid (MES) free acid monohydrate, 2-[4-(2-hydroxyethyl)piperazin-1-yl]ethanesulfonic acid (HEPES) sodium salt, HEPES free acid, EDTA disodium salt dehydrate, sodium chloride and ammonium acetate were purchased from Mandel Scientific Inc (Guelph, Ontario, Canada). SsoFast EvaGreen supermix was purchased from Bio-Rad for real-time PCR analysis. T4-DNA ligase, deoxynucleotide (dNTP) solution mix, Taq DNA polymerase with ThermoPol buffer, and low molecular weight DNA ladder were purchased from New England Biolabs. All metal ions, buffers and gel stock solutions were prepared with Milli-Q water.

In vitro selection. The initial DNA library was prepared by ligating Lib-FAM-N 35 (200 pmol) with LibrA (300 pmol) using the splint DNA (300 pmol). These DNAs were first mixed in buffer A (50 mM pH 7.5 Tris- $\mathrm{HCl}, \mathrm{pH} 7.5,10 \mathrm{mM} \mathrm{MgCl} 2$ ) and annealed at $95{ }^{\circ} \mathrm{C}$ for $1 \mathrm{~min}$ followed by slow cooling to room temperature. The T4 ligation protocol provided by New England Biolabs was followed. The ligated DNA product was purified with $10 \%$ denaturing polyacrylamide gel (dPAGE) and the ligated DNA was extracted from the gel with buffer B (1 mM EDTA, $10 \mathrm{mM}$ Tris-HCl, pH 7.0). The extracted DNA library was further concentrated via ethanol precipitation and re-suspended in $60 \mu \mathrm{L}$ of buffer C (50 mM MES, pH 6.0, $25 \mathrm{mM} \mathrm{NaCl}$ ), which was the selection buffer. This DNA was used directly as the DNA library for the first round of selection. For each of the subsequent rounds, the library was generated from PCR. For the cleavage step, the DNA pool was incubated with freshly prepared lanthanides. The 
incubation time and concentration of metal salts are in Table 1. For the last few rounds of selection, $\mathrm{Lu}^{3+}$ was added in $20 \mu \mathrm{M}$ incremental with $30 \mathrm{~min}$ intervals instead of all at once. This was to avoid its possible inhibition of DNAzyme activity at high concentration. After incubation, the reaction was quenched with $8 \mathrm{M}$ urea and was purified in 10\% dPAGE. A fraction of the selected DNA was extracted from the gel and further purified with a Sep-Pak C18 column (Waters). The purified selected DNA was then dried in an Eppendorf Vacufuge at $30{ }^{\circ} \mathrm{C}$ overnight. The dried DNA was re-suspended in $70 \mu \mathrm{L}$ of $5 \mathrm{mM}$ HEPES buffer (pH 7.5). The PCR conditions and cloning and sequencing were the same as described previously. ${ }^{39}$

Activity assays. Gel-based activity assays were performed with a final concentration of $0.7 \mu \mathrm{M}$ of the FAM-labeled substrate strand and $1.1 \mu \mathrm{M}$ of the enzyme. The DNAzyme complexes were prepared by annealing them in buffer $\mathrm{C}$ and a final of $10 \mu \mathrm{M}$ lanthanide ions were added to initiate the cleavage reaction. The products were separated on a denaturing polyacrylamide gel and analyzed using a Bio-Rad ChemiDoc MP imaging system.

Sensing. The sensing kinetics studies were carried out using 96 well plates and were monitored with Molecular Device SpectraMax M3 microplate reader. The complex was formed by annealing the FAMlabeled substrate and the quencher-labeled enzyme in buffer C. $100 \mu \mathrm{L}$ of $50 \mathrm{nM}$ FAM-Q DNAzyme in $1 \mathrm{mM}$ pH 7.5 HEPES (pH 7.5) was used for each well. $1 \mu \mathrm{L}$ of target ions was added after 5 min of background reading. The samples were continuously monitored after addition with $10 \mathrm{~s}$ interval.

\section{Results and Discussion}

In vitro selection. A total of 14 trivalent lanthanides are available for in vitro selection (the radioactive $\mathrm{Pm}^{3+}$ is excluded from discussion). Free lanthanide ions at high concentration (e.g. $5 \mathrm{mM}$ ) can cleave 
RNA efficiently, and previous studies indicated that larger lanthanides have much higher cleavage activity. ${ }^{41}$ To isolate highly active DNAzymes, we chose the largest lutetium $\left(\mathrm{Lu}^{3+}\right)$ for this in vitro selection experiment.

The next condition to consider is the DNA library design. Our previous selection was carried out with an N50 library (e.g. 50 random nucleotides). ${ }^{39}$ In that selection, a large fraction of the obtained sequences belong to the Ce13d (Figure 1E) family, which is similarly active with all trivalent lanthanide, including $\mathrm{Lu}^{3+}$. To discriminate different lanthanides, we need to avoid Ce13d. We noted that $\mathrm{Ce} 13 \mathrm{~d}$ is a relatively large enzyme and we might disfavor its appearance by using a smaller randomized region size. Therefore, instead of the N50 library, we chose an N35 library for this selection.

To isolate new lanthanide-specific DNAzymes, we started with an initial sequence population of $\sim 10^{14}$. The randomized region (the blue loop in Figure 1A) is flanked by two short base paired duplexes, holding the single ribo-adenosine (rA) to its proximity. This rA linkage serves as the putative cleavage site, since RNA is more susceptible to cleavage than DNA. ${ }^{42}$ The exact sequence of the library at this moment is shown in Figure 1B. The library was incubated with $\mathrm{Lu}^{3+}$ to induce cleavage (Figure 1A, step 1). After that, the shorter cleaved DNA strands were harvested using gel electrophoresis (step 2). Two rounds of polymerase chain reactions (PCR) were performed to bring the library back to the original length and amplify the cleaved DNA to seed the next round of selection (see Table S1 in SI for primer sequences). After 6 rounds of selection, cleavage reached $43 \%$ (See Table 1 for selection conditions and progress). At that point, the library was cloned and sequenced. 

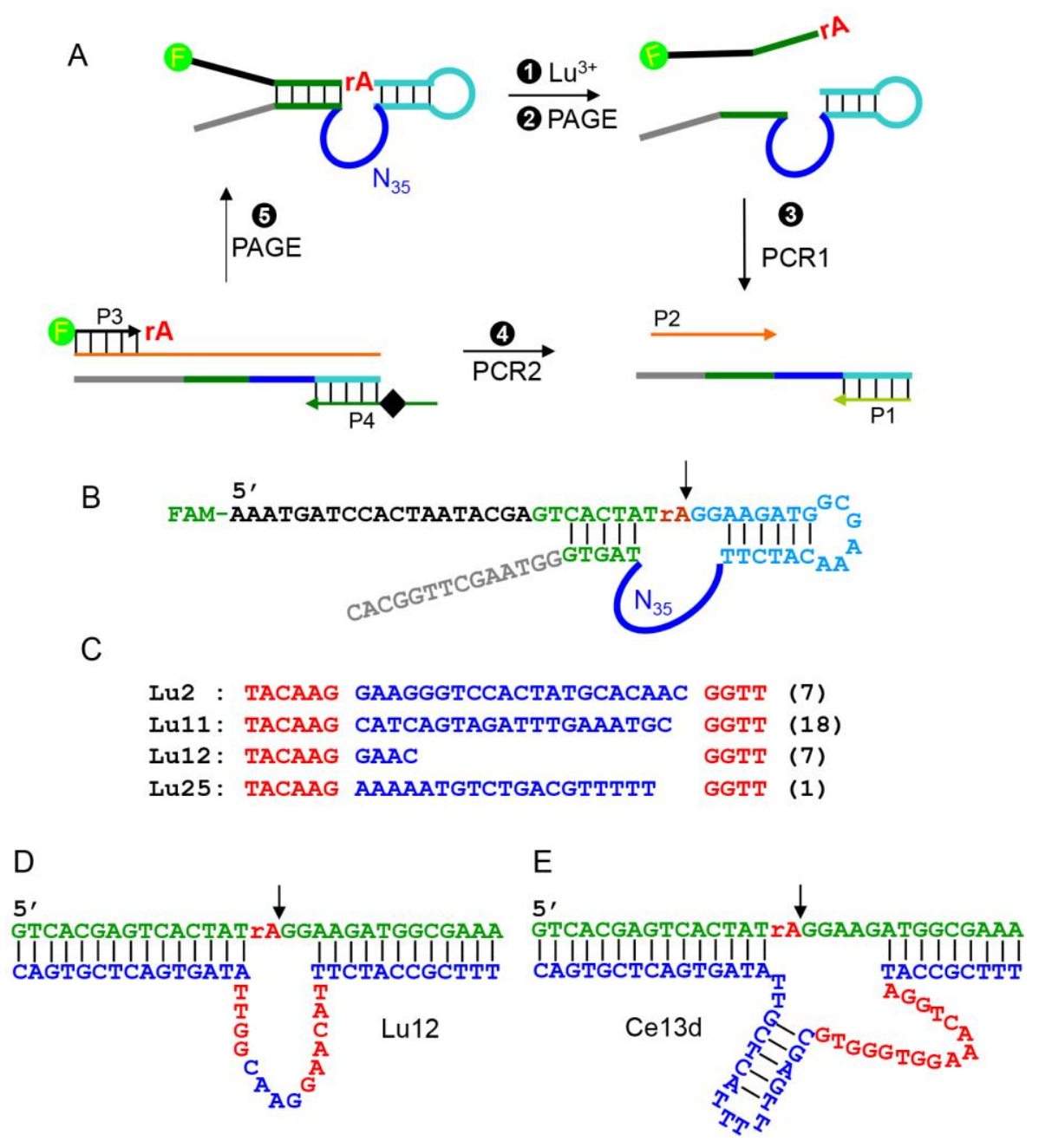

Figure 1. (A) Scheme of in vitro DNAzyme selection in the presence of $\mathrm{Lu}^{3+}$. The initial library contains an N35 randomized region and a single RNA linkage (rA) as the cleavage site. Cleaved sequences in the presence of $\mathrm{Lu}^{3+}$ (step 1) are harvested after gel electrophoresis (PAGE, step 2). After two rounds of PCR the full-length single-stranded library is re-generated and the positive strand is isolated after another PAGE step. (B) The library sequence before the cleavage step. (C) Sequence alignment from the start of the N35 region. The nucleotides in red are highly conserved. The numbers in the parenthesis are the number of identical or very similar sequences (differ by less than 2 nucleotides) as the listed. The secondary structure of the trans-cleaving Lu12 (D) and Ce13d (E) DNAzymes. Important nucleotides for catalysis are marked in red. 
We obtained a total of 38 valid sequences (see Table S2 for the full sequences). Interestingly, the Ce13d type of sequences were completely eliminated. All the selected DNA can be aligned into a single sequence family and some of the representative ones are listed in Figure 1C starting from the 5'-end of the random region. The first six nucleotides are highly conserved (TACAAAG, in red), followed by a highly variable region both in terms of sequence and length, and ended with another highly conserved tetra-nucleotide, GGTT. Since Lu12 has the shortest insertion between the two fixed regions, we folded it using mfold (Figure S1), ${ }^{43}$ and it can be easily engineered into a trans-cleaving version (Figure 1D). The two conserved regions are positioned at the two sides of this enzyme (in red). For those DNAzymes with longer insertions, the inserted sequences either form a hairpin or a structureless loop, suggesting that they might not participate in the catalytic reaction. One example of Lu1 folding is given in Figure S2, showing a larger loop.

Table 1. In vitro selection conditions and progress for $\mathrm{Lu}^{3+}$.

\begin{tabular}{llll}
\hline Round \# & {$\left[\mathbf{L u}^{3+}\right](\boldsymbol{\mu M})$} & Incubation time (min) & Cleavage (\%) \\
1 & 50 & 60 & 0.1 \\
2 & 50 & 60 & 0.2 \\
3 & 50 & 80 & 1.3 \\
4 & 50 & 80 & 21 \\
5 & 20 & 80 & 19 \\
6 & 20 & 80 & 43 \\
\hline
\end{tabular}


DNAzyme characterization. The structure of Lu12 is quite different from that of Ce13d (Figure 1E) and it might have a different activity trend cross the lanthanide series. For sensing applications, we are interested in its metal specificity. Therefore, its activity against other divalent and trivalent cations was first tested. With $10 \mu \mathrm{M}$ metal ions (Figure $2 \mathrm{~A}$ ), only $\mathrm{Y}^{3+}$ showed substantial cleavage and $\mathrm{Pb}^{2+}$ was moderately active. With $100 \mu \mathrm{M}$ concentration (Figure 2B), both $\mathrm{Pb}^{2+}$ and $\mathrm{Y}^{3+}$ showed a large fraction of cleavage. A smeared band was observed in the presence of $\mathrm{Au}^{3+}$, which might be due to DNA base binding but no cleavage was observed. $\mathrm{Pb}^{2+}$ is a very common interference for DNAzyme-based sensors. Fortunately, for lanthanide detection, the $\mathrm{Pb}^{2+}$ activity can be masked by adding thiol such as mercaptohexanol $(\mathrm{MCH}) .{ }^{39} \mathrm{Y}^{3+}$ interferes because of its similar size and charge to the lanthanides. The other 19 metal ions did not produce significant cleavage at both concentrations, suggesting this DNAzyme might be highly selective for lanthanides.

We next studied its activity as a function of lanthanide atomic number. After 5 min reaction with $1 \mu \mathrm{M}$ lanthanides (Figure 2C), cleavage was observed with all the samples. It is interesting to note that the smaller lanthanides (up to $\mathrm{Tb}^{3+}$ ) showed a similarly high efficiency of cleavage and the efficiency gradually decayed for the last few larger ones (Figure 2C). It is interesting to note that even though the selection was carried out with $\mathrm{Lu}^{3+}$ and only a single family of DNAzyme was obtained, $\mathrm{Lu}^{3+}$ gave the least cleavage. Next, we studied the enzyme activity as a function of metal concentration (Figure 2D). $\mathrm{Nd}^{3+}$ and $\mathrm{Lu}^{3+}$ were chosen to represent small and large lanthanide, respectively. Efficient cleavage was achieved when the metal concentration was from $0.5 \mu \mathrm{M}$ to a few $\mu \mathrm{M}$ for both metals. Inhibition occurred at higher metal concentrations, which can be explained by lanthanide non-specifically interacting with the DNAzyme. $\mathrm{Nd}^{3+}$ starts to inhibit activity at more than $10 \mu \mathrm{M}$ while $\mathrm{Lu}^{3+}$ inhibits at more than $2 \mu \mathrm{M}$. 
We next measured the cleavage rate (Figure 2E). With $10 \mu \mathrm{M} \mathrm{Nd}^{3+}$, a gradual increase of the cleaved fraction was observed with time and the rate was calculated to be $0.12 \mathrm{~min}^{-1}$. For comparison, $\mathrm{Lu}^{3+}$ barely induced any cleavage due to its inhibition effect at this concentration. For sensing applications, we should work with low lanthanide concentrations to avoid artifacts associated with DNAzyme activity inhibition.

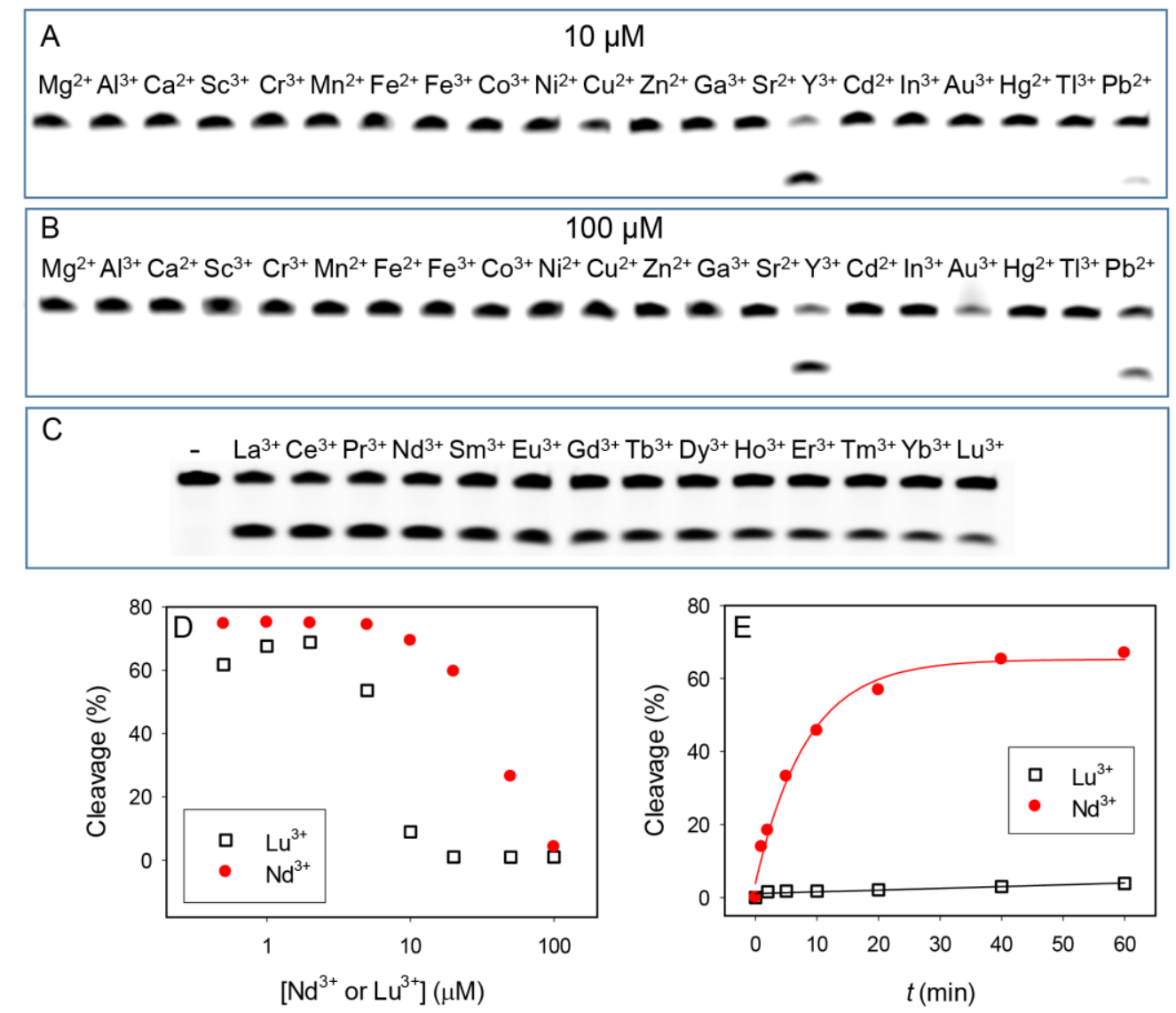

Figure 2. Gel images of the Lu12 DNAzyme assay with (A) $10 \mu \mathrm{M}$ and (B) $100 \mu \mathrm{M}$ metal ion for $1 \mathrm{~h}$. (C) Lu12 reacting with $1 \mu \mathrm{M}$ lanthanides for $5 \mathrm{~min}$. All assays are performed in $50 \mathrm{mM}$ MES buffer (pH 6.0) with $25 \mathrm{mM} \mathrm{NaCl}$. (D) Fraction of cleavage as a function of $\mathrm{Nd}^{3+}$ or $\mathrm{Lu}^{3+}$ concentration after $1 \mathrm{~h}$ reaction time. (D) Kinetics of Lu12 in the presence of $10 \mu \mathrm{M} \mathrm{Nd}^{3+}$ or $\mathrm{Lu}^{3+}$. 
Our selection results are interesting in the sense that $\mathrm{Lu}^{3+}$ gives the slowest activity among all the tested lanthanides even though the selection was carried out with $\mathrm{Lu}^{3+}$. With a high concentration of 5 mM lanthanides (no DNAzyme added), Matsumura and Komiyama reported that $\mathrm{Lu}^{3+}$ was among the most efficient lanthanide for cleaving a dinucleotide RNA while small lanthanides such as $\mathrm{Nd}^{3+}$ are almost inactive. ${ }^{41}$ On the other hand, Geyer and Sen reported that with $60 \mu \mathrm{M}$ lanthanides, $\mathrm{Lu}^{3+}$ has the lowest activity for cleaving a chimeric substrate alone (no DNAzyme). ${ }^{38}$ The activity is however very low (e.g. below $0.0012 \mathrm{~h}^{-1}$ ). When hybridized to the GR5 DNAzyme, which is highly active with $\mathrm{Pb}^{2+}$, $\mathrm{Lu}^{3+}$ induced the fastest cleavage. ${ }^{38} \mathrm{We}$ did most of our assays at even lower lanthanide concentrations (e.g. below $10 \mu \mathrm{M}$ in most cases) and found that $\mathrm{Lu}^{3+}$ was the least active.

It seems that lanthanide concentration, substrate length, and the presence of DNAzyme are all important to determine the activity trend. This led us to study lanthanide size-dependent cleavage of the substrate strand (30-mer, chimeric substrate) alone with a high lanthanide concentration. We incubated the substrate with $2 \mathrm{mM}$ lanthanides for $2.5 \mathrm{~h}$ (Figure 3A). Only $4 \%$ cleavage was observed for all the samples and the difference across the series is very small (Figure 3B). This inefficient cleavage is attributed to the condensation of the substrate by the high concentration of trivalent lanthanides, which may have shielded the RNA linkage from further attacks. Our results indicate that we should assay DNAzymes at low lanthanide concentrations to avoid denaturation. Under such conditions, the intrinsic RNA cleavage activity of lanthanides is very weak and DNAzymes can significantly accelerate it.

RNA cleavage has been extensively studied and both nucleobases and metal ions can participate the critical catalytic step. ${ }^{44}$ A generally proposed mechanism for metal ion is to act as a general base to assist deprotonation of the $2^{\prime}-\mathrm{OH}$ on the ribose. The $p \mathrm{~K}_{\mathrm{a}}$ value of lanthanide bound water ranges from 8.2 to 9.4 and larger lanthanides have lower $p \mathrm{~K}_{\mathrm{a}}$ values. Therefore, the bound water on $\mathrm{Lu}^{3+}$ deprotonates the most easily and should be the best general base catalyst. Since this does not explain our 
observation, besides the general base role, lanthanides must have additional roles such as neutralize the phosphate negative charges in the transition state, where the smaller lanthanides might be more effective. Detailed mechanistic studies will be a topic of follow-up research.

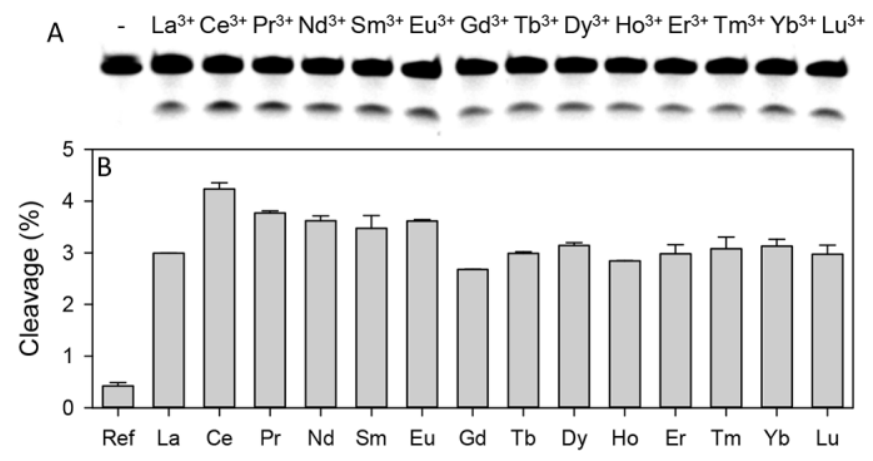

Figure 3. Gel image (A) and its quantification (B) of the FAM-labeled substrate strand (no enzyme strand) cleavage by $2 \mathrm{mM}$ lanthanides in MOPS buffer ( $\mathrm{pH} 7.0,50 \mathrm{mM}$ ), $25 \mathrm{mM} \mathrm{NaCl}$ for $2.5 \mathrm{~h}$. The first lane of the gel is a reference without lanthanide.

Overall, Lu12 is a DNAzyme highly specific for lanthanides, but it shows some lanthanide sizedependent activity that might be of analytically useful. Lu12 is the smallest DNAzyme within this family. It has a quite simple structure. To further understand its property, we next varied the size of the enzyme loop. Lu1 has a much larger loop (Figure S2), and it is also active with various lanthanides (Figure S3). We then truncated the loop size of Lu1 to produce two smaller DNAzymes (Lu1a and Lu1b), both are still active (Figure S4). Finally, we inserted a stretch of poly-A, poly-T and poly-C in the loop region and only the poly-C insertion inactivated the DNAzyme (Figure S5). These studies confirmed the secondary structure of Lu12. As long as the conserved nucleotides are maintained, the DNAzyme is likely to be still active. 
Lanthanide sensing. After understanding the structure and activity of the Lu12 DNAzyme, we next aim to test its performance as a biosensor. Since it shares the common secondary structure as most other RNA-cleaving DNAzymes, the catalytic beacon strategy was employed. ${ }^{45}$ The $3^{\prime}$-end of the substrate strand was labeled with a FAM (carboxyfluorescein) and the 5'-end of the enzyme was labeled with a dark quencher (Figure S6). This complex has low fluorescence in this initial state due to the proximity of the two labels (Figure 5A). Addition of $\mathrm{Nd}^{3+}$ produced $\sim 10$-fold fluorescence enhancement (Figure 4A) and the rate of enhancement was faster with higher $\mathrm{Nd}^{3+}$ concentrations. From these kinetic traces, the initial slope was plotted (Figure 4B). The Lu12 response can fit to binding to one $\mathrm{Nd}^{3+}$ ion with an apparent dissociation constant $\left(K_{\mathrm{d}}\right)$ of $38 \mathrm{nM}$. The initial response was linear (inset) and the limit of detection was determined to be $0.5 \mathrm{nM} \mathrm{Nd}^{3+}$ (or 72 parts-per-trillion) based on signal greater than three times of background variation. The selectivity test is similar to the results from the gel, where only $\mathrm{Y}^{3+}$ and $\mathrm{Pb}^{2+}$ produced response besides the lanthanide $\mathrm{Nd}^{3+}$ (Figure 4C). Within the lanthanide group, we observed quite different slopes and $\mathrm{Lu}^{3+}$ again produced the slowest response (Figure 4D). Therefore, the sensor is highly selective for lanthanides and the response decreases for the larger lanthanides. 

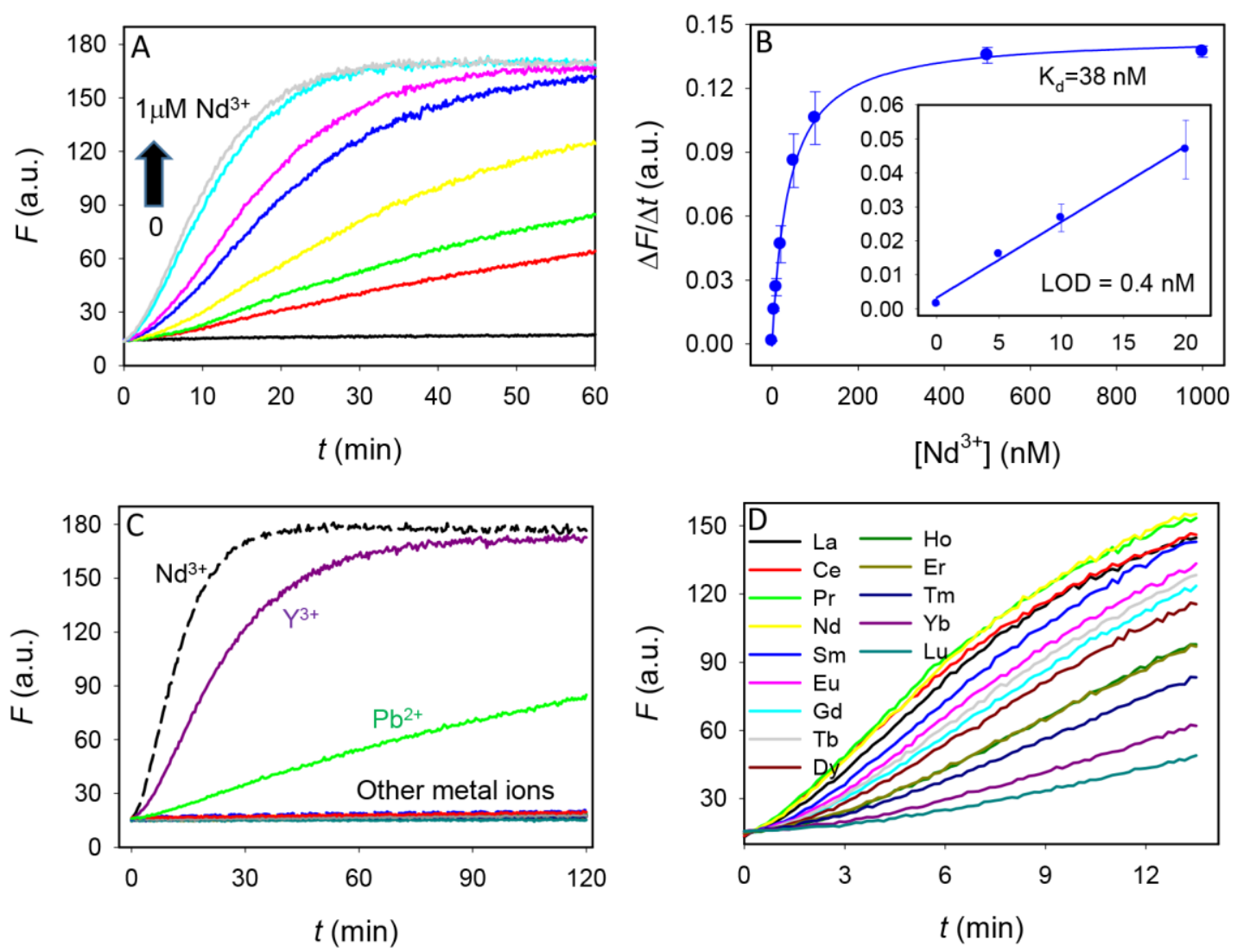

Figure 4. (A) Sensor signaling kinetics in the presence of various concentrations of $\mathrm{Nd}^{3+}$. DNAzyme sensor concentration $=50 \mathrm{nM}$. (B) Quantification of $\mathrm{Nd}^{3+}$ based on the initial rate of sensor fluorescence enhancement. Inset: the initial linear response at low $\mathrm{Nd}^{3+}$ concentrations. (C) Sensor response to 0.5 $\mu \mathrm{M}$ of divalent and trivalent metal ions. The list of the other metal ions tested can be found in Figure $2 \mathrm{~B}$. (D) Sensor response to $0.5 \mu \mathrm{M}$ of various lanthanides.

Ratiometric sensing. Now we have two lanthanide-dependent DNAzymes (Figure 1D, E). Both are highly specific to lanthanide. Next we aim to test whether we can extract more analytical information by combining the two DNAzymes (Figure 5A, B). In separate wells, we tested the response of each sensor to different lanthanides. For example, Figure 5C shows the rate of fluorescence change is very similar to 
$\mathrm{Nd}^{3+}$. On the other hand, $\mathrm{Lu}^{3+}$ produced much slower response with Lu12 than with Ce13d (Figure 5D). This is also consistent with our previous gel-based assays. Using these two sensors, different lanthanides may produce different sensor response patterns, which can be used for their identification. With this observation, the simplest mathematic treatment is ratiometric detection. The ratio of sensor signaling rate is plotted for all the lanthanides (Figure 5E). The ratio is close to 1 for lanthanides smaller than $\mathrm{Tb}^{3+}$, while for the larger ones, the ratio shows a descending trend.

Ideally, each lanthanide should have a unique ratio. However, since there are a total of 14 analytes and we only have two sensor probes, we cannot identify individual lanthanides with the current system. It is however encouraging that the larger lanthanides have a size-dependent response. To fully identify each lanthanide, it is likely that more sensor probes with unique patterns of response are needed.

A

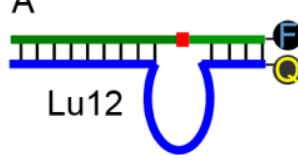

$\downarrow \mathrm{Ln}^{3+}$
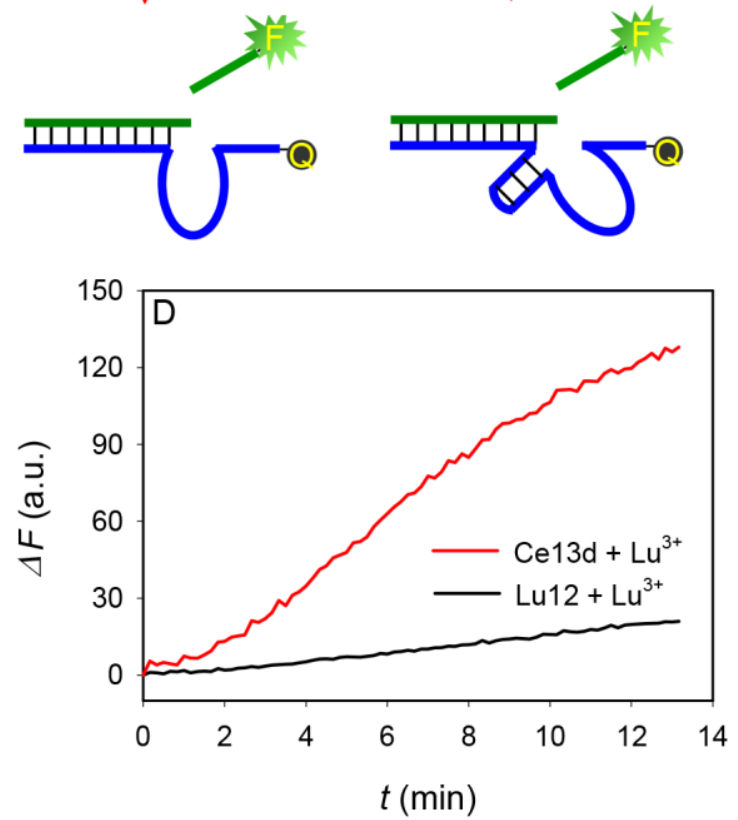

B

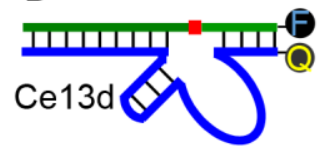

$\downarrow \mathrm{Ln}^{3+}$
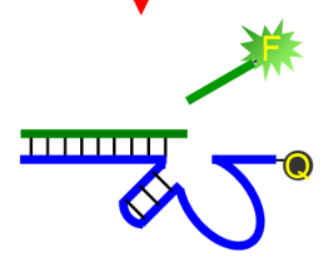
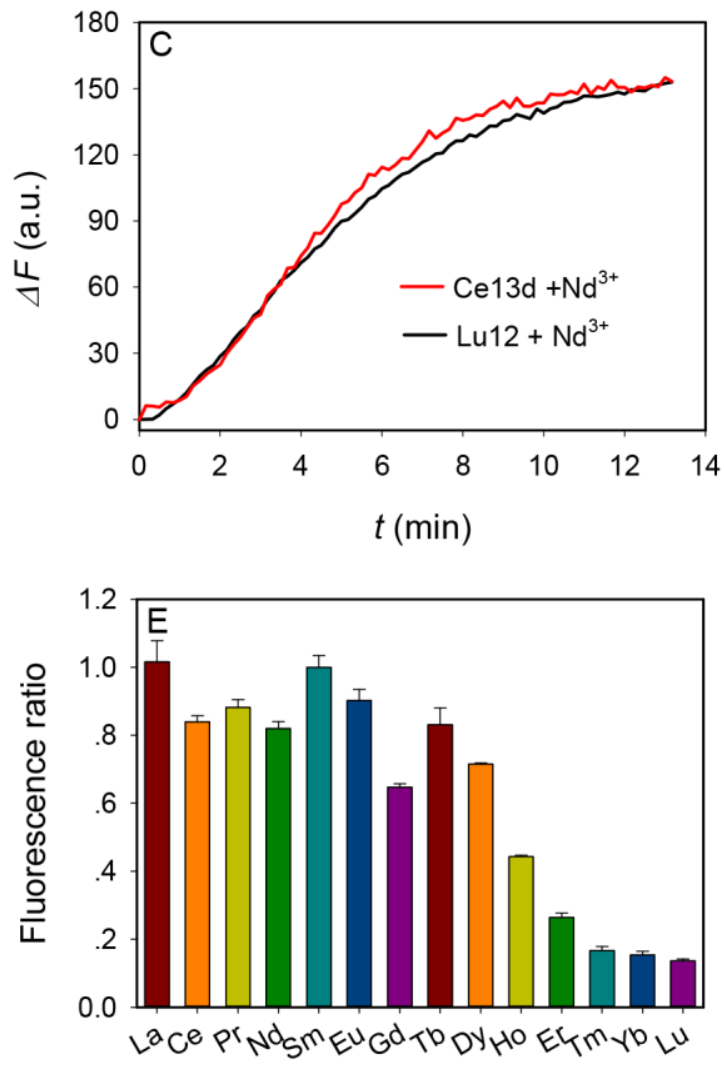
Figure 5. Schematics of detecting lanthanides using (A) the Lu12 DNAzyme and (B) the Ce13d DNAzyme based catalytic beacons. Sensor signal increase as a function of time for the two sensors in the presence of (C) $500 \mathrm{nM} \mathrm{Nd}^{3+}$ or (D) $500 \mathrm{nM} \mathrm{Lu}^{3+}$. (E) Ratio of fluorescence increase rate of the Lu12 over the Ce13d DNAzyme sensor.

Conclusions. In summary, we performed a DNAzyme in vitro selection experiment with $\mathrm{Lu}^{3+}$ as the metal cofactor. A new RNA-cleaving DNAzyme was obtained, which is active with lanthanides alone without the need of divalent metal ions. Importantly, it shows a lanthanide size dependent activity trend, providing a scaffold to study lanthanide coordination to DNA. Using two DNAzyme-based sensors, we can partially discriminate a few large lanthanides. With more unique DNA sequences obtained via in vitro selection, we might be able to form a larger sensor array to discriminate each lanthanide.

\section{Acknowledgements}

Funding for this work was from the University of Waterloo, Ontario Ministry of Research \& Innovation, and the Natural Sciences and Engineering Research Council (NSERC) of Canada (Discovery Grant and Strategic Project Grant).

Supporting Information. DNAzyme sequence alignment and characterization. "This material is available free of charge via the Internet at http://pubs.acs.org." 


\section{References}

(1) Kobayashi, S.; Sugiura, M.; Kitagawa, H.; Lam, W. W. L. Chem. Rev. 2002, 102, 2227-2302.

(2) Wang, F.; Han, Y.; Lim, C. S.; Lu, Y. H.; Wang, J.; Xu, J.; Chen, H. Y.; Zhang, C.; Hong, M. H.; Liu, X. G. Nature 2010, 463, 1061-1065.

(3) Atwood, D. A. In The Rare Earth Elements: Fundamentals and Applications; Atwood, D. A., Ed.; Wiley, 2012, pp 21-25.

(4) Houk, R. S.; Fassel, V. A.; Flesch, G. D.; Svec, H. J.; Gray, A. L.; Taylor, C. E. Anal. Chem. 1980, 52, 2283-2289.

(5) Vargas-Zuniga, G. I.; Sessler, J. L. In The Rare Earth Elements: Fundamentals and Applications; Atwood, D. A., Ed.; Wiley, 2012, pp 561-573.

(6) Mercier, D.; Leconte, N.; Methivier, C.; Suzenet, F.; Guillaumet, G.; Wuillaume, A.; Pradier, C.M. Phys. Chem. Chem. Phys. 2010, 12, 6099-6106.

(7) Saito, S.; Hoshino, H. Anal. Bioanal. Chem. 2004, 378, 1644-1647.

(8) Kolarik, Z. Chem. Rev. 2008, 108, 4208-4252.

(9) Nishiyabu, R.; Hashimoto, N.; Cho, T.; Watanabe, K.; Yasunaga, T.; Endo, A.; Kaneko, K.; Niidome, T.; Murata, M.; Adachi, C.; Katayama, Y.; Hashizume, M.; Kimizuka, N. J. Am. Chem. Soc. 2009, 131, 2151-2158.

(10) Wang, F.; Liu, B.; Huang, P.-J. J.; Liu, J. Anal. Chem. 2013, 85, 12144-12151.

(11) Zhang, M.; Le, H.-N.; Jiang, X.-Q.; Yin, B.-C.; Ye, B.-C. Anal. Chem. 2013, 85, 11665-11674.

(12) Lin, W. T. D.; Huang, P.-J. J.; Pautler, R.; Liu, J. Chem. Commun. 2014, DOI: 10.1039/C4CC05551A.

(13) Kim, H.-K.; Li, J.; Nagraj, N.; Lu, Y. Chem. Eur. J. 2008, 14, 8696-8703.

(14) Feig, A. L.; Panek, M.; Horrocks, W. D., Jr; Uhlenbeck, O. C. Chem. Biol. 1999, 6, 801-810. 
(15) Marciniec, T.; Ciesiołka, J.; Wrzesinski, J.; Krzyżosiak, W. J. FEBS Lett. 1989, 243, 293-298.

(16) Breaker, R. R. Nat. Biotechnol. 1997, 15, 427-431.

(17) Santoro, S. W.; Joyce, G. F. Proc. Natl. Acad. Sci. U.S.A. 1997, 94, 4262-4266.

(18) Navani, N. K.; Li, Y. Curr. Opin. Chem. Biol. 2006, 10, 272-281.

(19) Lu, Y. Chem. Eur. J. 2002, 8, 4588-4596.

(20) Wilson, D. S.; Szostak, J. W. Annu. Rev. Biochem. 1999, 68, 611-647.

(21) Silverman, S. K. Acc. Chem. Res. 2009, 42, 1521-1531.

(22) Liu, J.; Cao, Z.; Lu, Y. Chem. Rev. 2009, 109, 1948-1998.

(23) Zhang, X.-B.; Kong, R.-M.; Lu, Y. Annu. Rev. Anal. Chem. 2011, 4, 105-128.

(24) Liu, J.; Lu, Y. J. Am. Chem. Soc. 2003, 125, 6642-6643.

(25) Xiao, Y.; Rowe, A. A.; Plaxco, K. W. J. Am. Chem. Soc. 2007, 129, 262.

(26) Liu, Z.; Mei, S. H. J.; Brennan, J. D.; Li, Y. J. Am. Chem. Soc. 2003, 125, 7539-7545.

(27) Lu, L. M.; Zhang, X. B.; Kong, R. M.; Yang, B.; Tan, W. H. J. Am. Chem. Soc. 2011, 133, 11686-11691.

(28) Wang, H.; Kim, Y.; Liu, H.; Zhu, Z.; Bamrungsap, S.; Tan, W. J. Am. Chem. Soc. 2009, 131, 8221-8226.

(29) Breaker, R. R.; Joyce, G. F. Chem. Biol. 1994, 1, 223-229.

(30) Li, J.; Zheng, W.; Kwon, A. H.; Lu, Y. Nucleic Acids Res. 2000, 28, 481-488.

(31) Carmi, N.; Balkhi, H. R.; Breaker, R. R. Proc. Natl. Acad. Sci. U.S.A. 1998, 95, 2233-2237.

(32) Liu, J.; Brown, A. K.; Meng, X.; Cropek, D. M.; Istok, J. D.; Watson, D. B.; Lu, Y. Proc. Natl. Acad. Sci. U.S.A. 2007, 104, 2056-2061.

(33) Hollenstein, M.; Hipolito, C.; Lam, C.; Dietrich, D.; Perrin, D. M. Angew. Chem., Int. Ed. 2008, 47, $4346-4350$. 
(34) Dokukin, V.; Silverman, S. K. Chem. Sci. 2012, 3, 1707-1714.

(35) Javadi-Zarnaghi, F.; Hobartner, C. J. Am. Chem. Soc. 2013, 135, 12839-12848.

(36) Sugimoto, N.; Ohmichi, T. FEBS Lett. 1996, 393, 97-100.

(37) Feig, A. L.; Scott, W. G.; Uhlenbeck, O. C. Science 1998, 279, 81-84.

(38) Geyer, C. R.; Sen, D. J.Mol.Biol. 1998, 275, 483-489.

(39) Huang, P.-J. J.; Lin, J.; Cao, J.; Vazin, M.; Liu, J. Anal. Chem. 2014, 86, 1816-1821.

(40) Komiyama, M.; Takeda, N.; Shigekawa, H. Chem. Commun. 1999, 1443-1451.

(41) Matsumura, K.; Komiyama, M. J. Biochem. 1997, 122, 387-394.

(42) Li, Y.; Breaker, R. R. J. Am. Chem. Soc. 1999, 121, 5364-5372.

(43) Zuker, M. Nucleic Acids Res. 2003, 31, 3406-3415.

(44) Ward, W. L.; Plakos, K.; DeRose, V. J. Chem. Rev. 2014, 114, 4318-4342.

(45) Li, J.; Lu, Y. J. Am. Chem. Soc. 2000, 122, 10466-10467. 